Acta Crystallographica Section E

Structure Reports

Online

ISSN 1600-5368

\section{4-(4-Carboxy-1,3-thiazol-2-yl)pyridinium 3-carboxy-4-hydroxybenzenesulfonate dihydrate}

\section{Zhong-Xiang Du* and Jun-Xia Li}

Department of Chemistry and Chemical Engineering, Luoyang Normal University, Luoyang, Henan 471022, People's Republic of China

Correspondence e-mail: dzx6281@126.com

Received 1 September 2008; accepted 25 September 2008

Key indicators: single-crystal X-ray study; $T=291 \mathrm{~K}$; mean $\sigma(\mathrm{C}-\mathrm{C})=0.002 \AA$; $R$ factor $=0.032 ; w R$ factor $=0.092 ;$ data-to-parameter ratio $=12.7$.

In the crystal structure of the title compound, $\mathrm{C}_{9} \mathrm{H}_{7} \mathrm{~N}_{2} \mathrm{O}_{2} \mathrm{~S}^{+}$.$\mathrm{C}_{7} \mathrm{H}_{5} \mathrm{O}_{6} \mathrm{~S}^{-} \cdot 2 \mathrm{H}_{2} \mathrm{O}$, an $\mathrm{H}$ atom from the 5-sulfosalicylic acid is transferred to the pyridyl $\mathrm{N}$ atom, forming a salt. The dihedral angle between the thiazole and pyridinium rings is $5.909(5)^{\circ}$. The crystal packing is determined by $\mathrm{O}-\mathrm{H} \cdots \mathrm{O}$ and $\mathrm{N}-$ $\mathrm{H}$... O hydrogen bonds involving water molecules.

\section{Related literature}

For related structures, see: Chen et al. (2007); Ellsworth et al. (2006); Su et al. (2004).<smiles>O=C(O)c1csc(-c2cc[nH+]cc2)n1</smiles><smiles>O=C(O)c1cc(S(=O)(=O)[O-])ccc1O</smiles>
$\cdot 2 \mathrm{H}_{2} \mathrm{O}$

\section{Experimental}

Crystal data

$\mathrm{C}_{9} \mathrm{H}_{7} \mathrm{~N}_{2} \mathrm{O}_{2} \mathrm{~S}^{+} \cdot \mathrm{C}_{7} \mathrm{H}_{5} \mathrm{O}_{6} \mathrm{~S}^{-} \cdot 2 \mathrm{H}_{2} \mathrm{O}$

$M_{r}=460.43$

Triclinic, $P \overline{1}$

$a=8.6234(14) \AA$

$b=10.6065(17) \AA$

$c=10.7979$ (17) $\AA$

$\alpha=97.799$ (2)

$\beta=94.479$ (2) $\gamma=99.885(2)^{\circ}$ $V=958.7(3) \AA^{3}$ $Z=2$

Mo $K \alpha$ radiation $\mu=0.34 \mathrm{~mm}^{-1}$ $T=291(2) \mathrm{K}$ $0.44 \times 0.29 \times 0.24 \mathrm{~mm}$
Data collection

Bruker APEXII CCD area-detector diffractometer

Absorption correction: multi-scan (SADABS; Sheldrick, 1996)

$T_{\min }=0.867, T_{\max }=0.924$

Refinement

$R\left[F^{2}>2 \sigma\left(F^{2}\right)\right]=0.032$

$w R\left(F^{2}\right)=0.092$

$S=1.03$

3494 reflections

275 parameters

Table 1

Hydrogen-bond geometry $\left(\AA,^{\circ}\right)$.

\begin{tabular}{|c|c|c|c|c|}
\hline$D-\mathrm{H} \cdots A$ & $D-\mathrm{H}$ & $\mathrm{H} \cdots A$ & $D \cdots A$ & $D-\mathrm{H} \cdots A$ \\
\hline $\mathrm{O} 1-\mathrm{H} 1 \cdots \mathrm{O} 2$ & 0.82 & 1.88 & $2.599(2)$ & 146 \\
\hline $\mathrm{O} 3-\mathrm{H} 3 \cdots \mathrm{O} 9$ & 0.82 & 1.71 & $2.5269(17)$ & 171 \\
\hline $\mathrm{O} 8-\mathrm{H} 8 \cdots \mathrm{O} 4^{\mathrm{i}}$ & 0.82 & 1.89 & $2.6979(18)$ & 171 \\
\hline $\mathrm{O} 9-\mathrm{H} 1 W \cdots \mathrm{O}^{\mathrm{ii}}$ & 0.84 & 1.93 & $2.753(2)$ & 165 \\
\hline $\mathrm{O} 9-\mathrm{H} 2 W \cdots \mathrm{O} 5^{\mathrm{iii}}$ & 0.83 & 1.89 & $2.713(2)$ & 172 \\
\hline $\mathrm{O} 10-\mathrm{H} 3 W \cdots \mathrm{O} 2$ & 0.81 & 2.32 & $2.9001(19)$ & 129 \\
\hline $\mathrm{O} 10-\mathrm{H} 4 W \cdots \mathrm{O} 7^{\mathrm{iv}}$ & 0.81 & 2.27 & $2.835(2)$ & 128 \\
\hline $\mathrm{N} 2-\mathrm{H} 2 D \cdots \mathrm{O} 10^{\mathrm{v}}$ & 0.86 & 1.86 & $2.689(2)$ & 162 \\
\hline
\end{tabular}

Symmetry codes: (i) $-x+1,-y,-z$; (ii) $x, y+1, z$; (iii) $-x,-y+1,-z+1$; (iv) $x, y+1, z+1 ;(\mathrm{v})-x+1,-y+2,-z+1$.

Data collection: APEX2 (Bruker, 2004); cell refinement: APEX2; data reduction: SAINT (Bruker, 2004); $\operatorname{program}(\mathrm{s})$ used to solve structure: SHELXS97 (Sheldrick, 2008); program(s) used to refine structure: SHELXL97 (Sheldrick, 2008); molecular graphics: SHELXTL (Sheldrick, 2008); software used to prepare material for publication: SHELXTL.

This work was supported by the National Natural Science Foundation of China (No. 20471026) and the Natural Science Foundation of Henan Province (No. 0311021200).

Supplementary data and figures for this paper are available from the IUCr electronic archives (Reference: KP2193).

\section{References}

Bruker (2004). APEX2 and SAINT. Bruker AXS Inc., Madison, Wisconsin, USA.

Chen, X. D., Wu, H. F., Zhao, X. H., Zhao, X. J. \& Du, M. (2007). Cryst. Growth Des. 7, 124-131.

Ellsworth, J. M., Su, C. Y., Khaliq, Z., Hipp, R. E., Goforth, A. M., Smith, M. D. \& Loye, H. C. (2006). J. Mol. Struct. 796, 86-94.

Sheldrick, G. M. (1996). SADABS. University of Göttingen, Germany.

Sheldrick, G. M. (2008). Acta Cryst. A64, 112-122.

Su, C. Y., Smith, M. D., Goforth, A. M. \& Loye, H. C. (2004). Inorg. Chem. 43, 6881-6883.
$\mathrm{H}$-atom parameters constrained

$\Delta \rho_{\max }=0.31{\mathrm{e} \AA^{-3}}^{-3}$

$\Delta \rho_{\min }=-0.29 \mathrm{e}^{-3}$ 3494 independent reflections 3095 reflections with $I>2 \sigma(I)$ $R_{\text {int }}=0.014$ 


\section{supporting information}

Acta Cryst. (2008). E64, o2044 [doi:10.1107/S1600536808030924]

\section{4-(4-Carboxy-1,3-thiazol-2-yl)pyridinium 3-carboxy-4-hydroxybenzenesulfonate dihydrate}

\section{Zhong-Xiang Du and Jun-Xia Li}

\section{S1. Comment}

2-(4-Pyridyl)thiazole-4-carboxylic acid (HPTCA), which is an asymmetric, chelating ligand, has been studied in recent years. Five of its transition metal complexes (Chen et al., 2007; Ellsworth et al., 2006; Su et al., 2004) were reported. In this paper we describe its salt with 5-sulfosalicylic acid ( $\left.\mathrm{H}_{3} \mathrm{SSA}\right)$, (I).

The crystal structure of the title molecule comprises 2-(4-pyridylomium)thiazole-4-carboxylic acid, a 5-sulfosalicylic acid anion and two water molecules (Fig.1). The $\mathrm{H}$ atom of the 5 -sulfosalicylic acid is transferred to the pyridyl $\mathrm{N}$-atom of 2-(4-pyridyl)thiazole-4-carboxylic acid, thus forming a salt. The dihedral angle between the thiazole and pyridinium rings is $5.909(5)^{\circ}$. The $\mathrm{N}-\mathrm{H}$ and $\mathrm{O}-\mathrm{H}$ groups are involved in intra- and intermolecular hydrogen bonds with water molecules generating the 3-dimensional hydrogen bond network (Table 1 and Fig. 2).

\section{S2. Experimental}

The ligand HPTCA ( $1 \mathrm{mmol}, 0.21 \mathrm{~g})$ and $\mathrm{H}_{3} \mathrm{SSA} .2 \mathrm{H}_{2} \mathrm{O}(1 \mathrm{mmol}, 0.25 \mathrm{~g})$ were dissolved in solvent mixture of water and methanol $\left(20 \mathrm{~mL}, v / v\right.$ 1:1). To this solution, $\mathrm{Cu}\left(\mathrm{CH}_{3} \mathrm{COO}\right)_{2} .4 \mathrm{H}_{2} \mathrm{O}(1 \mathrm{mmol}, 0.26 \mathrm{~g})$ was added and the resulting mixture was stirred and refluxed at $353 \mathrm{~K}$ for $3 \mathrm{~h}$, then cooled to room temperature. After filtration and evaporation in air for five days, colourless claviform-shaped crystals were obtained in a yield of 43\%. Analysis, found (\%): C, 41.75; H, 3.51; N, 6.02; $\mathrm{S}, 13.87 . \mathrm{C}_{16} \mathrm{H}_{16} \mathrm{~N}_{2} \mathrm{O}_{10} \mathrm{~S}_{2}$ requires (\%): $\mathrm{C}, 41.70 ; \mathrm{H}, 3.47 ; \mathrm{N}, 6.08 ; \mathrm{S}, 13.90$. (The elemental analysis indicates that the copper(II) is not coordinated by the ligands) (CCDC number 685021)

\section{S3. Refinement}

$\mathrm{H}$ Atoms bonded to $\mathrm{C}$ or $\mathrm{N}$ atoms were positioned geometrically with $\mathrm{C}-\mathrm{H}$ distance of $0.93 \AA$ and $\mathrm{N}-\mathrm{H}$ distance of 0.86 $\AA$, and treated as riding atoms, with $U_{\text {iso }}(\mathrm{H})=1.2 U_{\text {eq }}(\mathrm{C}$ or $\mathrm{N})$. $\mathrm{H}$ atoms bonded to $\mathrm{O}$ atoms were located in a difference Fourier map and refined isotropically. 

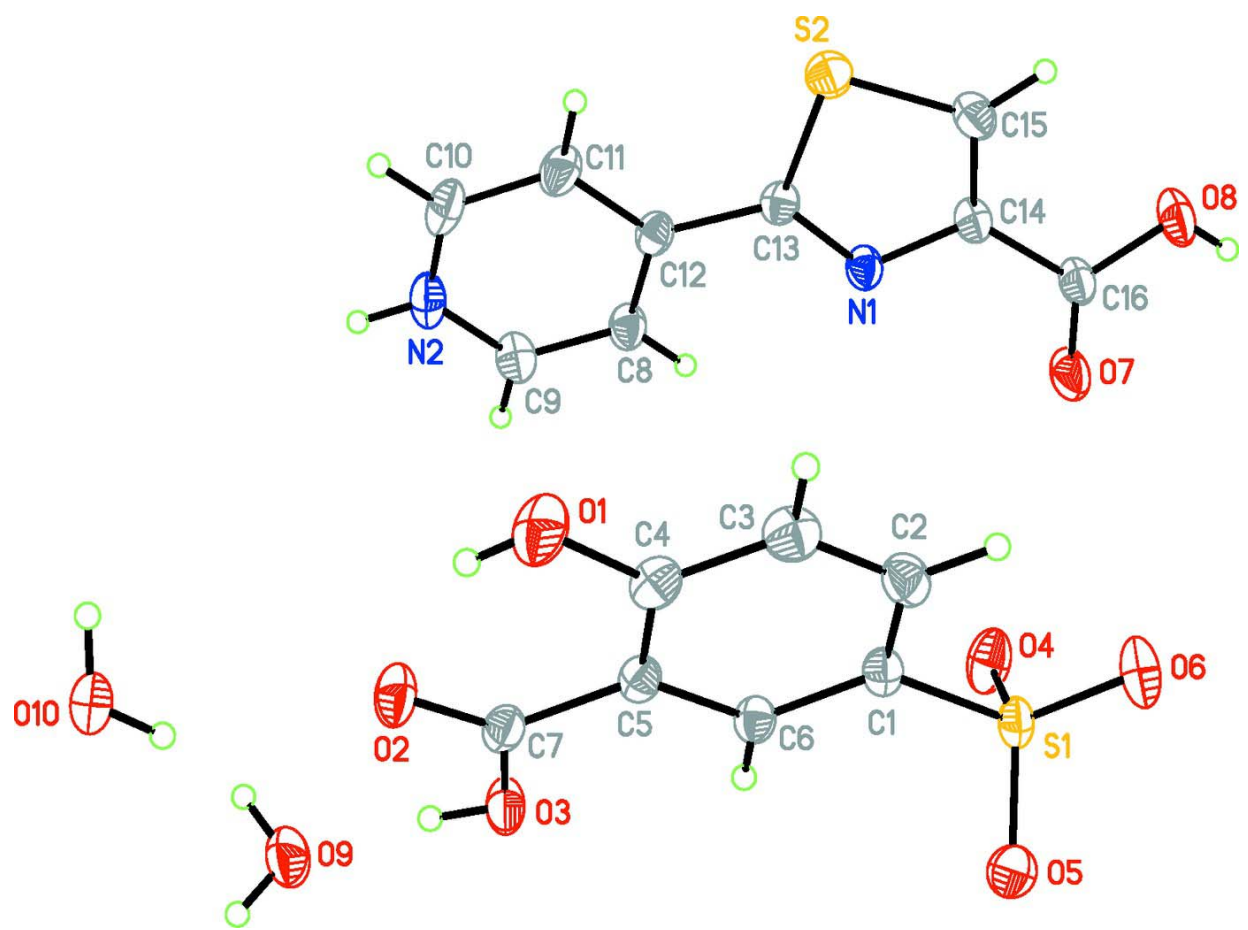

\section{Figure 1}

Molecular structure of (I), with displacement ellipsoids drawn at the $30 \%$ probability level.

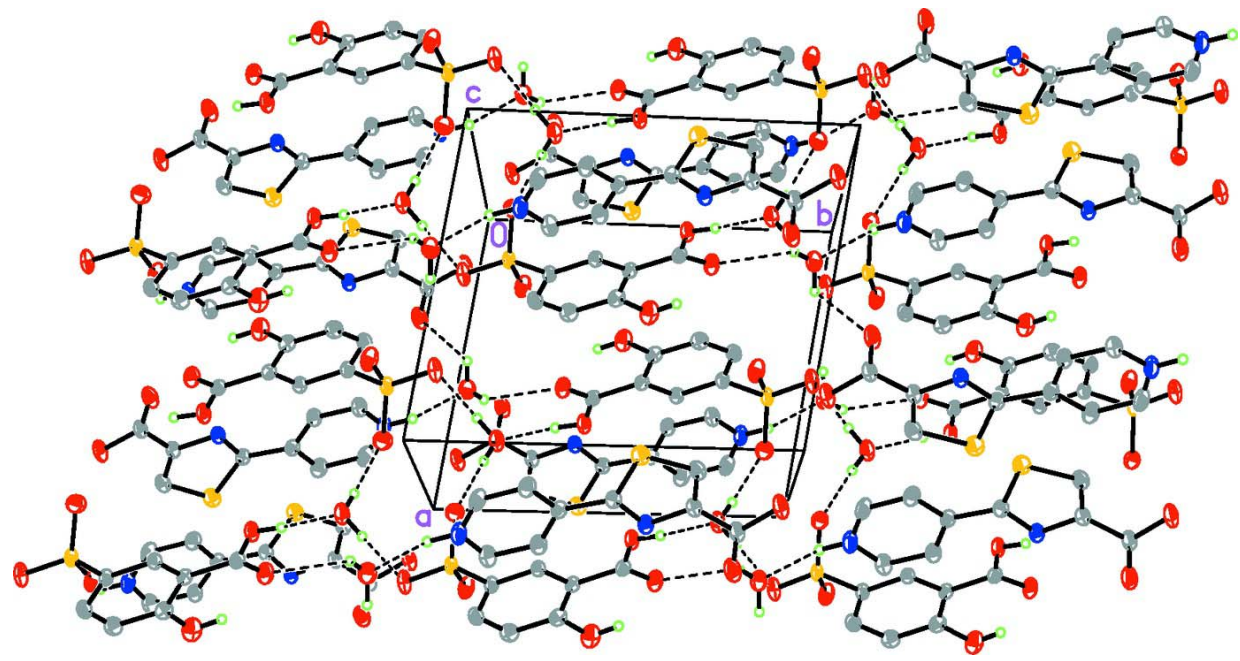

Figure 2

The crystal packing of (I), showing hydrogen bonds as dashed lines. For the sake of clarity, $\mathrm{H}$ atoms on $\mathrm{C}$ atoms have been omitted.

\section{4-(4-Carboxy-1,3-thiazol-2-yl)pyridinium 3-carboxy-4-hydroxybenzenesulfonate dihydrate}

Crystal data

$\mathrm{C}_{9} \mathrm{H}_{7} \mathrm{~N}_{2} \mathrm{O}_{2} \mathrm{~S}^{+} \cdot \mathrm{C}_{7} \mathrm{H}_{5} \mathrm{O}_{6} \mathrm{~S}^{-} \cdot 2 \mathrm{H}_{2} \mathrm{O}$

$M_{r}=460.43$

Triclinic, $P \overline{1}$

Hall symbol: -P 1

$$
\begin{aligned}
& a=8.6234(14) \AA \\
& b=10.6065(17) \AA \\
& c=10.7979(17) \AA \\
& \alpha=97.799(2)^{\circ}
\end{aligned}
$$


$\beta=94.479(2)^{\circ}$

$\gamma=99.885(2)^{\circ}$

$V=958.7(3) \AA^{3}$

$Z=2$

$F(000)=476$

$D_{\mathrm{x}}=1.595 \mathrm{Mg} \mathrm{m}^{-3}$

Mo $K \alpha$ radiation, $\lambda=0.71073 \AA$

Data collection

Bruker APEXII CCD area-detector diffractometer

Radiation source: fine-focus sealed tube

Graphite monochromator

$\varphi$ and $\omega$ scans

Absorption correction: multi-scan

(SADABS; Sheldrick, 1996)

$T_{\min }=0.867, T_{\max }=0.924$

Refinement

Refinement on $F^{2}$

Least-squares matrix: full

$R\left[F^{2}>2 \sigma\left(F^{2}\right)\right]=0.032$

$w R\left(F^{2}\right)=0.092$

$S=1.03$

3494 reflections

275 parameters

6 restraints

Primary atom site location: structure-invariant direct methods

Secondary atom site location: difference Fourier map

\section{Special details}

Geometry. All e.s.d.'s (except the e.s.d. in the dihedral angle between two 1.s. planes) are estimated using the full covariance matrix. The cell e.s.d.'s are taken into account individually in the estimation of e.s.d.'s in distances, angles and torsion angles; correlations between e.s.d.'s in cell parameters are only

used when they are defined by crystal symmetry. An approximate (isotropic)

treatment of cell e.s.d.'s is used for estimating e.s.d.'s involving 1.s. planes.

Refinement. Refinement of $F^{2}$ against ALL reflections. The weighted $R$-factor $w R$ and goodness of fit $S$ are based on $F^{2}$, conventional $R$-factors $R$ are based on $F$, with $F$ set to zero for negative $F^{2}$. The threshold expression of $F^{2}>\sigma\left(F^{2}\right)$ is used only for calculating $R$-factors(gt) etc. and is not relevant to the choice of reflections for refinement. $R$-factors based on $F^{2}$ are statistically about twice as large as those based on $F$, and $R$ factors based on ALL data will be even larger.

Fractional atomic coordinates and isotropic or equivalent isotropic displacement parameters $\left(\AA^{2}\right)$

\begin{tabular}{lllll}
\hline & $x$ & $y$ & $z$ & $U_{\text {iso }} * U_{\text {eq }}$ \\
\hline S1 & $0.25056(5)$ & $0.11285(4)$ & $0.35763(4)$ & $0.03667(14)$ \\
S2 & $1.01768(5)$ & $0.41512(5)$ & $0.19381(4)$ & $0.04482(15)$ \\
O1 & $0.52983(18)$ & $0.53943(14)$ & $0.76634(12)$ & $0.0532(4)$ \\
H1 & 0.4963 & 0.6058 & 0.7574 & $0.080^{*}$
\end{tabular}

Cell parameters from 4068 reflections

Claviform, colourless

7016 measured reflections

3494 independent reflections

$R_{\text {int }}=0.014$

$\theta_{\max }=25.5^{\circ}, \theta_{\min }=2.4^{\circ}$

$k=-12 \rightarrow 12$

$l=-13 \rightarrow 13$

Hydrogen site location: inferred from

-atom parameters constrained

where $P=\left(F_{\mathrm{o}}^{2}+2 F_{\mathrm{c}}^{2}\right) / 3$

$(\Delta / \sigma)_{\max }<0.001$

$\Delta \rho_{\max }=0.31 \mathrm{e}^{-3}$

$\Delta \rho_{\min }=-0.29$ e $\AA^{-3}$

Extinction correction: SHELXL97,

$\mathrm{Fc}^{*}=\mathrm{kFc}\left[1+0.001 \mathrm{xFc}^{2} \lambda^{3} / \sin (2 \theta)\right]^{-1 / 4}$

Extinction coefficient: 0.021 (2) $\mu=0.34 \mathrm{~mm}^{-1}$

0.080




\begin{tabular}{|c|c|c|c|c|}
\hline $\mathrm{O} 2$ & $0.34176(16)$ & $0.67695(12)$ & $0.67191(12)$ & $0.0472(3)$ \\
\hline $\mathrm{O} 3$ & $0.18259(15)$ & $0.59188(11)$ & $0.49653(12)$ & $0.0432(3)$ \\
\hline H3 & 0.1683 & 0.6667 & 0.4998 & $0.065^{*}$ \\
\hline $\mathrm{O} 4$ & $0.30140(18)$ & $0.15439(12)$ & $0.24126(12)$ & $0.0513(4)$ \\
\hline O5 & $0.07938(16)$ & $0.09571(14)$ & $0.35499(15)$ & 0.0595 (4) \\
\hline O6 & $0.31219(19)$ & $0.00026(13)$ & $0.38592(14)$ & $0.0577(4)$ \\
\hline $\mathrm{O} 7$ & $0.61923(18)$ & $0.12933(14)$ & $-0.10879(16)$ & $0.0643(4)$ \\
\hline O8 & 0.81979 (18) & $0.03591(13)$ & $-0.04892(13)$ & 0.0525 (4) \\
\hline H8 & 0.7748 & -0.0241 & -0.1027 & $0.079 *$ \\
\hline O9 & $0.11825(18)$ & $0.81397(13)$ & $0.48548(15)$ & $0.0566(4)$ \\
\hline $\mathrm{H} 1 \mathrm{~W}$ & 0.1851 & 0.8757 & 0.4688 & $0.085^{*}$ \\
\hline $\mathrm{H} 2 \mathrm{~W}$ & 0.0648 & 0.8431 & 0.5397 & $0.085^{*}$ \\
\hline $\mathrm{O} 10$ & $0.35251(19)$ & $0.94663(14)$ & $0.77184(18)$ & $0.0746(5)$ \\
\hline $\mathrm{H} 3 \mathrm{~W}$ & 0.3066 & 0.8901 & 0.7160 & $0.112^{*}$ \\
\hline $\mathrm{H} 4 \mathrm{~W}$ & 0.4477 & 0.9618 & 0.7742 & $0.112^{*}$ \\
\hline N1 & $0.77420(17)$ & $0.36608(13)$ & $0.03035(13)$ & 0.0356 \\
\hline N2 & $0.7588(2)$ & $0.83610(15)$ & $0.15551(16)$ & $0.0488(4)$ \\
\hline $\mathrm{H} 2 \mathrm{D}$ & 0.7349 & 0.9120 & 0.1660 & $0.059^{*}$ \\
\hline $\mathrm{C} 1$ & $0.33096(19)$ & $0.24035(15)$ & $0.48162(15)$ & $0.0324(4)$ \\
\hline $\mathrm{C} 2$ & $0.4457(2)$ & $0.22332(18)$ & $0.57360(17)$ & $0.0398(4)$ \\
\hline $\mathrm{H} 2$ & 0.4793 & 0.1444 & 0.5709 & $0.048^{*}$ \\
\hline $\mathrm{C} 3$ & $0.5089(2)$ & $0.32448(19)$ & $0.66860(17)$ & $0.0428(4)$ \\
\hline $\mathrm{H} 3 \mathrm{~A}$ & 0.5849 & 0.3130 & 0.7300 & $0.051^{*}$ \\
\hline $\mathrm{C} 4$ & $0.4600(2)$ & $0.44306(17)$ & $0.67308(15)$ & $0.0368(4)$ \\
\hline $\mathrm{C} 5$ & $0.34238(19)$ & $0.46005(15)$ & $0.58262(14)$ & $0.0310(3)$ \\
\hline C6 & $0.27899(19)$ & $0.35698(15)$ & $0.48679(15)$ & $0.0312(3)$ \\
\hline H6 & 0.2013 & 0.3672 & 0.4261 & $0.037^{*}$ \\
\hline $\mathrm{C} 7$ & $0.28871(19)$ & $0.58539(16)$ & $0.58768(15)$ & $0.0336(4)$ \\
\hline $\mathrm{C} 8$ & $0.7104(2)$ & $0.62363(17)$ & $0.04650(16)$ & $0.0396(4)$ \\
\hline $\mathrm{H} 8 \mathrm{~A}$ & 0.6538 & 0.5612 & -0.0173 & $0.048^{*}$ \\
\hline $\mathrm{C} 9$ & $0.6759(2)$ & $0.74537(19)$ & $0.06439(18)$ & $0.0473(5)$ \\
\hline H9 & 0.5946 & 0.7655 & 0.0132 & $0.057^{*}$ \\
\hline $\mathrm{C} 10$ & $0.8772(3)$ & $0.81171(18)$ & $0.23019(19)$ & $0.0517(5)$ \\
\hline H10 & 0.9334 & 0.8769 & 0.2917 & $0.062^{*}$ \\
\hline $\mathrm{C} 11$ & $0.9166(2)$ & $0.69063(18)$ & $0.21681(18)$ & $0.0456(4)$ \\
\hline H11 & 0.9993 & 0.6738 & 0.2688 & $0.055^{*}$ \\
\hline $\mathrm{C} 12$ & $0.8314(2)$ & $0.59344(16)$ & $0.12459(15)$ & $0.0342(4)$ \\
\hline $\mathrm{C} 13$ & $0.8626(2)$ & $0.46044(16)$ & $0.10910(15)$ & $0.0336(4)$ \\
\hline $\mathrm{C} 14$ & $0.8288(2)$ & $0.25269(16)$ & $0.03559(15)$ & $0.0354(4)$ \\
\hline $\mathrm{C} 15$ & $0.9591(2)$ & $0.26100(18)$ & $0.11859(17)$ & $0.0420(4)$ \\
\hline H15 & 1.0088 & 0.1921 & 0.1320 & $0.050^{*}$ \\
\hline $\mathrm{C} 16$ & $0.7430(2)$ & $0.13402(17)$ & $-0.04781(17)$ & $0.0402(4)$ \\
\hline
\end{tabular}

Atomic displacement parameters $\left(\AA^{2}\right)$

\begin{tabular}{lllllll}
\hline & $U^{11}$ & $U^{22}$ & $U^{33}$ & $U^{12}$ & $U^{13}$ & $U^{23}$ \\
\hline S1 & $0.0437(3)$ & $0.0231(2)$ & $0.0418(3)$ & $0.00772(17)$ & $0.00411(18)$ & $-0.00162(17)$ \\
S2 & $0.0427(3)$ & $0.0475(3)$ & $0.0405(3)$ & $0.0097(2)$ & $-0.01003(19)$ & $0.0000(2)$
\end{tabular}




$\begin{array}{lllllll}\text { O1 } & 0.0644(9) & 0.0472(8) & 0.0389(7) & 0.0037(7) & -0.0181(6) & -0.0035(6) \\ \text { O2 } & 0.0590(8) & 0.0326(7) & 0.0432(7) & 0.0049(6) & -0.0022(6) & -0.0095(6) \\ \text { O3 } & 0.0501(7) & 0.0283(6) & 0.0480(7) & 0.0111(5) & -0.0082(6) & -0.0028(5) \\ \text { O4 } & 0.0753(10) & 0.0361(7) & 0.0368(7) & 0.0011(6) & 0.0047(6) & -0.0024(5) \\ \text { O5 } & 0.0440(8) & 0.0457(8) & 0.0774(10) & 0.0017(6) & 0.0034(7) & -0.0207(7) \\ \text { O6 } & 0.0805(10) & 0.0317(7) & 0.0647(9) & 0.0235(7) & 0.0058(8) & 0.0046(6) \\ \text { O7 } & 0.0581(9) & 0.0427(8) & 0.0821(11) & 0.0191(7) & -0.0240(8) & -0.0207(7) \\ \text { O8 } & 0.0696(9) & 0.0377(7) & 0.0499(8) & 0.0260(7) & -0.0084(7) & -0.0067(6) \\ \text { O9 } & 0.0660(9) & 0.0342(7) & 0.0736(10) & 0.0169(6) & 0.0145(7) & 0.0081(7) \\ \text { O10 } & 0.0556(9) & 0.0383(8) & 0.1174(14) & 0.0034(7) & 0.0108(9) & -0.0263(8) \\ \text { N1 } & 0.0414(8) & 0.0307(7) & 0.0332(7) & 0.0090(6) & -0.0031(6) & 0.0001(6) \\ \text { N2 } & 0.0659(11) & 0.0289(8) & 0.0519(10) & 0.0092(7) & 0.0134(8) & 0.0022(7) \\ \text { C1 } & 0.0370(9) & 0.0281(8) & 0.0322(8) & 0.0062(7) & 0.0051(7) & 0.0034(7) \\ \text { C2 } & 0.0419(10) & 0.0378(9) & 0.0430(10) & 0.0134(8) & 0.0039(8) & 0.0106(8) \\ \text { C3 } & 0.0404(10) & 0.0505(11) & 0.0380(9) & 0.0094(8) & -0.0043(7) & 0.0126(8) \\ \text { C4 } & 0.0390(9) & 0.0404(9) & 0.0278(8) & 0.0010(7) & 0.0002(7) & 0.0039(7) \\ \text { C5 } & 0.0338(8) & 0.0298(8) & 0.0281(8) & 0.0026(7) & 0.0052(6) & 0.0028(6) \\ \text { C6 } & 0.0342(8) & 0.0295(8) & 0.0285(8) & 0.0053(6) & -0.0008(6) & 0.0027(6) \\ \text { C7 } & 0.0359(8) & 0.0297(8) & 0.0321(8) & 0.0002(7) & 0.0055(7) & 0.0003(7) \\ \text { C8 } & 0.0465(10) & 0.0344(9) & 0.0352(9) & 0.0076(8) & -0.0003(7) & -0.0023(7) \\ \text { C9 } & 0.0567(12) & 0.0403(10) & 0.0457(11) & 0.0141(9) & 0.0041(9) & 0.0033(8) \\ \text { C10 } & 0.0633(13) & 0.0330(10) & 0.0494(11) & -0.0050(9) & 0.0027(10) & -0.0075(8) \\ \text { C11 } & 0.0500(11) & 0.0388(10) & 0.0412(10) & 0.0005(8) & -0.0061(8) & -0.0026(8) \\ \text { C12 } & 0.0398(9) & 0.0319(9) & 0.0286(8) & 0.0022(7) & 0.0049(7) & 0.0009(7) \\ \text { C13 } & 0.0374(9) & 0.0341(9) & 0.0277(8) & 0.0054(7) & 0.0006(7) & 0.0024(7) \\ \text { C14 } & 0.0416(9) & 0.0334(9) & 0.0325(8) & 0.0118(7) & 0.0026(7) & 0.0036(7) \\ \text { C15 } & 0.0448(10) & 0.0421(10) & 0.0411(10) & 0.0171(8) & 0.0002(8) & 0.0043(8) \\ \text { C16 } & 0.0475(10) & 0.0329(9) & 0.0407(10) & 0.0147(8) & 0.0007(8) & -0.0001(7)\end{array}$

Geometric parameters $\left(\AA,{ }^{\circ}\right)$

\begin{tabular}{llll}
\hline $\mathrm{S} 1-\mathrm{O} 6$ & $1.4469(14)$ & $\mathrm{C} 1-\mathrm{C} 6$ & $1.382(2)$ \\
$\mathrm{S} 1-\mathrm{O} 5$ & $1.4535(14)$ & $\mathrm{C} 1-\mathrm{C} 2$ & $1.398(2)$ \\
$\mathrm{S} 1-\mathrm{O} 4$ & $1.4613(14)$ & $\mathrm{C} 2-\mathrm{C} 3$ & 0.9300 \\
$\mathrm{~S} 1-\mathrm{C} 1$ & $1.7731(17)$ & $\mathrm{C} 2-\mathrm{H} 2$ & $1.390(3)$ \\
$\mathrm{S} 2-\mathrm{C} 15$ & $1.6968(19)$ & $\mathrm{C} 3-\mathrm{C} 4$ & 0.9300 \\
$\mathrm{~S} 2-\mathrm{C} 13$ & $1.7334(17)$ & $\mathrm{C} 3-\mathrm{H} 3 \mathrm{~A}$ & $1.404(2)$ \\
$\mathrm{O} 1-\mathrm{C} 4$ & $1.355(2)$ & $\mathrm{C} 4-\mathrm{C} 5$ & $1.401(2)$ \\
$\mathrm{O} 1-\mathrm{H} 1$ & 0.8200 & $\mathrm{C} 5-\mathrm{C} 6$ & $1.476(2)$ \\
$\mathrm{O} 2-\mathrm{C} 7$ & $1.234(2)$ & $\mathrm{C} 5-\mathrm{C} 7$ & 0.9300 \\
$\mathrm{O} 3-\mathrm{C} 7$ & $1.308(2)$ & $\mathrm{C} 6-\mathrm{H} 6$ & $1.366(3)$ \\
$\mathrm{O} 3-\mathrm{H} 3$ & 0.8200 & $\mathrm{C} 8-\mathrm{C} 9$ & $1.397(2)$ \\
$\mathrm{O} 7-\mathrm{C} 16$ & $\mathrm{C} 8-\mathrm{C} 12$ & 0.9300 \\
$\mathrm{O} 8-\mathrm{C} 16$ & $1.199(2)$ & $\mathrm{C} 8-\mathrm{H} 8 \mathrm{~A}$ & 0.9300 \\
$\mathrm{O} 8-\mathrm{H} 8$ & $1.325(2)$ & $\mathrm{C} 9-\mathrm{H} 9$ & $1.376(3)$ \\
$\mathrm{O} 9-\mathrm{H} 1 \mathrm{~W}$ & 0.8200 & $\mathrm{C} 10-\mathrm{C} 11$ & 0.9300 \\
$\mathrm{O} 9-\mathrm{H} 2 \mathrm{~W}$ & 0.8436 & $\mathrm{C} 10-\mathrm{H} 10$ & $1.393(2)$ \\
$\mathrm{O} 10-\mathrm{H} 3 \mathrm{~W}$ & 0.8319 & $\mathrm{C} 11-\mathrm{C} 12$ &
\end{tabular}




\begin{tabular}{|c|c|c|c|}
\hline $\mathrm{O} 10-\mathrm{H} 4 \mathrm{~W}$ & 0.8065 & $\mathrm{C} 11-\mathrm{H} 11$ & 0.9300 \\
\hline $\mathrm{N} 1-\mathrm{C} 13$ & $1.308(2)$ & $\mathrm{C} 12-\mathrm{C} 13$ & $1.471(2)$ \\
\hline $\mathrm{N} 1-\mathrm{C} 14$ & $1.371(2)$ & $\mathrm{C} 14-\mathrm{C} 15$ & $1.365(2)$ \\
\hline $\mathrm{N} 2-\mathrm{C} 10$ & $1.333(3)$ & $\mathrm{C} 14-\mathrm{C} 16$ & $1.487(2)$ \\
\hline $\mathrm{N} 2-\mathrm{C} 9$ & $1.342(3)$ & $\mathrm{C} 15-\mathrm{H} 15$ & 0.9300 \\
\hline $\mathrm{N} 2-\mathrm{H} 2 \mathrm{D}$ & 0.8600 & & \\
\hline $\mathrm{O} 6-\mathrm{S} 1-\mathrm{O} 5$ & $113.03(9)$ & $\mathrm{C} 1-\mathrm{C} 6-\mathrm{H} 6$ & 119.7 \\
\hline $\mathrm{O} 6-\mathrm{S} 1-\mathrm{O} 4$ & $112.45(9)$ & $\mathrm{C} 5-\mathrm{C} 6-\mathrm{H} 6$ & 119.7 \\
\hline $\mathrm{O} 5-\mathrm{S} 1-\mathrm{O} 4$ & $110.37(9)$ & $\mathrm{O} 2-\mathrm{C} 7-\mathrm{O} 3$ & $122.98(16)$ \\
\hline $\mathrm{O} 6-\mathrm{S} 1-\mathrm{C} 1$ & $106.53(8)$ & $\mathrm{O} 2-\mathrm{C} 7-\mathrm{C} 5$ & $121.86(16)$ \\
\hline $\mathrm{O} 5-\mathrm{S} 1-\mathrm{C} 1$ & $106.90(8)$ & $\mathrm{O} 3-\mathrm{C} 7-\mathrm{C} 5$ & $115.17(14)$ \\
\hline $\mathrm{O} 4-\mathrm{S} 1-\mathrm{C} 1$ & $107.15(8)$ & $\mathrm{C} 9-\mathrm{C} 8-\mathrm{C} 12$ & $119.66(17)$ \\
\hline $\mathrm{C} 15-\mathrm{S} 2-\mathrm{C} 13$ & $89.46(8)$ & $\mathrm{C} 9-\mathrm{C} 8-\mathrm{H} 8 \mathrm{~A}$ & 120.2 \\
\hline $\mathrm{C} 4-\mathrm{O} 1-\mathrm{H} 1$ & 109.5 & $\mathrm{C} 12-\mathrm{C} 8-\mathrm{H} 8 \mathrm{~A}$ & 120.2 \\
\hline $\mathrm{C} 7-\mathrm{O} 3-\mathrm{H} 3$ & 109.5 & $\mathrm{~N} 2-\mathrm{C} 9-\mathrm{C} 8$ & $120.27(18)$ \\
\hline $\mathrm{C} 16-\mathrm{O} 8-\mathrm{H} 8$ & 109.5 & $\mathrm{~N} 2-\mathrm{C} 9-\mathrm{H} 9$ & 119.9 \\
\hline $\mathrm{H} 1 \mathrm{~W}-\mathrm{O} 9-\mathrm{H} 2 \mathrm{~W}$ & 108.7 & $\mathrm{C} 8-\mathrm{C} 9-\mathrm{H} 9$ & 119.9 \\
\hline $\mathrm{H} 3 \mathrm{~W}-\mathrm{O} 10-\mathrm{H} 4 \mathrm{~W}$ & 115.9 & $\mathrm{~N} 2-\mathrm{C} 10-\mathrm{C} 11$ & $120.40(17)$ \\
\hline $\mathrm{C} 13-\mathrm{N} 1-\mathrm{C} 14$ & $110.35(14)$ & $\mathrm{N} 2-\mathrm{C} 10-\mathrm{H} 10$ & 119.8 \\
\hline $\mathrm{C} 10-\mathrm{N} 2-\mathrm{C} 9$ & $121.80(17)$ & $\mathrm{C} 11-\mathrm{C} 10-\mathrm{H} 10$ & 119.8 \\
\hline $\mathrm{C} 10-\mathrm{N} 2-\mathrm{H} 2 \mathrm{D}$ & 119.1 & $\mathrm{C} 10-\mathrm{C} 11-\mathrm{C} 12$ & $119.39(18)$ \\
\hline $\mathrm{C} 9-\mathrm{N} 2-\mathrm{H} 2 \mathrm{D}$ & 119.1 & $\mathrm{C} 10-\mathrm{C} 11-\mathrm{H} 11$ & 120.3 \\
\hline $\mathrm{C} 6-\mathrm{C} 1-\mathrm{C} 2$ & $120.15(15)$ & $\mathrm{C} 12-\mathrm{C} 11-\mathrm{H} 11$ & 120.3 \\
\hline $\mathrm{C} 6-\mathrm{C} 1-\mathrm{S} 1$ & $119.39(12)$ & $\mathrm{C} 11-\mathrm{C} 12-\mathrm{C} 8$ & $118.45(16)$ \\
\hline $\mathrm{C} 2-\mathrm{C} 1-\mathrm{S} 1$ & $120.46(13)$ & $\mathrm{C} 11-\mathrm{C} 12-\mathrm{C} 13$ & $122.13(16)$ \\
\hline $\mathrm{C} 3-\mathrm{C} 2-\mathrm{C} 1$ & $119.70(16)$ & $\mathrm{C} 8-\mathrm{C} 12-\mathrm{C} 13$ & $119.41(15)$ \\
\hline $\mathrm{C} 3-\mathrm{C} 2-\mathrm{H} 2$ & 120.2 & $\mathrm{~N} 1-\mathrm{C} 13-\mathrm{C} 12$ & $122.45(15)$ \\
\hline $\mathrm{C} 1-\mathrm{C} 2-\mathrm{H} 2$ & 120.2 & $\mathrm{~N} 1-\mathrm{C} 13-\mathrm{S} 2$ & $114.42(13)$ \\
\hline $\mathrm{C} 2-\mathrm{C} 3-\mathrm{C} 4$ & $120.60(16)$ & $\mathrm{C} 12-\mathrm{C} 13-\mathrm{S} 2$ & $123.13(12)$ \\
\hline $\mathrm{C} 2-\mathrm{C} 3-\mathrm{H} 3 \mathrm{~A}$ & 119.7 & $\mathrm{~N} 1-\mathrm{C} 14-\mathrm{C} 15$ & $115.53(16)$ \\
\hline $\mathrm{C} 4-\mathrm{C} 3-\mathrm{H} 3 \mathrm{~A}$ & 119.7 & $\mathrm{~N} 1-\mathrm{C} 14-\mathrm{C} 16$ & $118.19(14)$ \\
\hline $\mathrm{O} 1-\mathrm{C} 4-\mathrm{C} 3$ & $117.74(15)$ & $\mathrm{C} 15-\mathrm{C} 14-\mathrm{C} 16$ & $126.27(16)$ \\
\hline $\mathrm{O} 1-\mathrm{C} 4-\mathrm{C} 5$ & $122.23(16)$ & $\mathrm{C} 14-\mathrm{C} 15-\mathrm{S} 2$ & $110.25(13)$ \\
\hline $\mathrm{C} 3-\mathrm{C} 4-\mathrm{C} 5$ & $120.04(16)$ & $\mathrm{C} 14-\mathrm{C} 15-\mathrm{H} 15$ & 124.9 \\
\hline $\mathrm{C} 4-\mathrm{C} 5-\mathrm{C} 6$ & $118.92(15)$ & $\mathrm{S} 2-\mathrm{C} 15-\mathrm{H} 15$ & 124.9 \\
\hline $\mathrm{C} 4-\mathrm{C} 5-\mathrm{C} 7$ & $120.28(15)$ & $\mathrm{O} 7-\mathrm{C} 16-\mathrm{O} 8$ & $124.15(17)$ \\
\hline $\mathrm{C} 6-\mathrm{C} 5-\mathrm{C} 7$ & $120.79(15)$ & $\mathrm{O} 7-\mathrm{C} 16-\mathrm{C} 14$ & $123.19(16)$ \\
\hline $\mathrm{C} 1-\mathrm{C} 6-\mathrm{C} 5$ & $120.57(15)$ & $\mathrm{O} 8-\mathrm{C} 16-\mathrm{C} 14$ & $112.65(15)$ \\
\hline $\mathrm{O} 6-\mathrm{S} 1-\mathrm{C} 1-\mathrm{C} 6$ & $-173.04(13)$ & $\mathrm{C} 12-\mathrm{C} 8-\mathrm{C} 9-\mathrm{N} 2$ & $0.8(3)$ \\
\hline $\mathrm{O} 5-\mathrm{S} 1-\mathrm{C} 1-\mathrm{C} 6$ & $-51.93(16)$ & $\mathrm{C} 9-\mathrm{N} 2-\mathrm{C} 10-\mathrm{C} 11$ & $-0.9(3)$ \\
\hline $\mathrm{O} 4-\mathrm{S} 1-\mathrm{C} 1-\mathrm{C} 6$ & $66.39(15)$ & $\mathrm{N} 2-\mathrm{C} 10-\mathrm{C} 11-\mathrm{C} 12$ & $-0.1(3)$ \\
\hline $\mathrm{O} 6-\mathrm{S} 1-\mathrm{C} 1-\mathrm{C} 2$ & $6.85(17)$ & $\mathrm{C} 10-\mathrm{C} 11-\mathrm{C} 12-\mathrm{C} 8$ & $1.5(3)$ \\
\hline $\mathrm{O} 5-\mathrm{S} 1-\mathrm{C} 1-\mathrm{C} 2$ & $127.96(15)$ & $\mathrm{C} 10-\mathrm{C} 11-\mathrm{C} 12-\mathrm{C} 13$ & $-177.15(17)$ \\
\hline $\mathrm{O} 4-\mathrm{S} 1-\mathrm{C} 1-\mathrm{C} 2$ & $-113.72(15)$ & $\mathrm{C} 9-\mathrm{C} 8-\mathrm{C} 12-\mathrm{C} 11$ & $-1.8(3)$ \\
\hline $\mathrm{C} 6-\mathrm{C} 1-\mathrm{C} 2-\mathrm{C} 3$ & $-1.1(3)$ & $\mathrm{C} 9-\mathrm{C} 8-\mathrm{C} 12-\mathrm{C} 13$ & $176.87(16)$ \\
\hline $\mathrm{S} 1-\mathrm{C} 1-\mathrm{C} 2-\mathrm{C} 3$ & $179.01(13)$ & $\mathrm{C} 14-\mathrm{N} 1-\mathrm{C} 13-\mathrm{C} 12$ & $-179.49(15)$ \\
\hline
\end{tabular}




$-0.3(3)$
$-178.07(16)$
$1.7(3)$
$178.11(15)$
$-1.6(2)$
$-0.9(3)$
$179.36(15)$
$1.1(2)$
$-178.97(12)$
$0.2(2)$
$179.24(14)$
$-1.7(2)$
$179.26(15)$
$177.84(15)$
$-1.2(2)$
$0.6(3)$

$-0.3(3)$

$\mathrm{C} 2-\mathrm{C} 3-\mathrm{C} 4-\mathrm{O} 1$

$\mathrm{C} 2-\mathrm{C} 3-\mathrm{C} 4-\mathrm{C} 5$

$\mathrm{O} 1-\mathrm{C} 4-\mathrm{C} 5-\mathrm{C} 6$

$\mathrm{C} 3-\mathrm{C} 4-\mathrm{C} 5-\mathrm{C} 6$

$\mathrm{O} 1-\mathrm{C} 4-\mathrm{C} 5-\mathrm{C} 7$

$\mathrm{C} 3-\mathrm{C} 4-\mathrm{C} 5-\mathrm{C} 7$

$\mathrm{C} 2-\mathrm{C} 1-\mathrm{C} 6-\mathrm{C} 5$

$\mathrm{S} 1-\mathrm{C} 1-\mathrm{C} 6-\mathrm{C} 5$

$\mathrm{C} 4-\mathrm{C} 5-\mathrm{C} 6-\mathrm{C} 1$

$\mathrm{C} 7-\mathrm{C} 5-\mathrm{C} 6-\mathrm{C} 1$

$\mathrm{C} 4-\mathrm{C} 5-\mathrm{C} 7-\mathrm{O} 2$

$\mathrm{C} 6-\mathrm{C} 5-\mathrm{C} 7-\mathrm{O} 2$

$\mathrm{C} 4-\mathrm{C} 5-\mathrm{C} 7-\mathrm{O} 3$

$\mathrm{C} 6-\mathrm{C} 5-\mathrm{C} 7-\mathrm{O} 3$

$\mathrm{C} 10-\mathrm{N} 2-\mathrm{C} 9-\mathrm{C} 8$

$$
\begin{aligned}
& \mathrm{C} 14-\mathrm{N} 1-\mathrm{C} 13-\mathrm{S} 2 \\
& \mathrm{C} 11-\mathrm{C} 12-\mathrm{C} 13-\mathrm{N} 1 \\
& \mathrm{C} 8-\mathrm{C} 12-\mathrm{C} 13-\mathrm{N} 1 \\
& \mathrm{C} 11-\mathrm{C} 12-\mathrm{C} 13-\mathrm{S} 2 \\
& \mathrm{C} 8-\mathrm{C} 12-\mathrm{C} 13-\mathrm{S} 2 \\
& \mathrm{C} 15-\mathrm{S} 2-\mathrm{C} 13-\mathrm{N} 1 \\
& \mathrm{C} 15-\mathrm{S} 2-\mathrm{C} 13-\mathrm{C} 12 \\
& \mathrm{C} 13-\mathrm{N} 1-\mathrm{C} 14-\mathrm{C} 15 \\
& \mathrm{C} 13-\mathrm{N} 1-\mathrm{C} 14-\mathrm{C} 16 \\
& \mathrm{~N} 1-\mathrm{C} 14-\mathrm{C} 15-\mathrm{S} 2 \\
& \mathrm{C} 16-\mathrm{C} 14-\mathrm{C} 15-\mathrm{S} 2 \\
& \mathrm{C} 13-\mathrm{S} 2-\mathrm{C} 15-\mathrm{C} 14 \\
& \mathrm{~N} 1-\mathrm{C} 14-\mathrm{C} 16-\mathrm{O} 7 \\
& \mathrm{C} 15-\mathrm{C} 14-\mathrm{C} 16-\mathrm{O} 7 \\
& \mathrm{~N} 1-\mathrm{C} 14-\mathrm{C} 16-\mathrm{O} 8 \\
& \mathrm{C} 15-\mathrm{C} 14-\mathrm{C} 16-\mathrm{O} 8
\end{aligned}
$$

Hydrogen-bond geometry $\left(\AA,{ }^{\circ}\right)$

\begin{tabular}{lllll}
\hline$D-\mathrm{H} \cdots A$ & $D-\mathrm{H}$ & $\mathrm{H} \cdots A$ & $D \cdots A$ & $D-\mathrm{H} \cdots A$ \\
\hline $\mathrm{O} 1-\mathrm{H} 1 \cdots \mathrm{O} 2$ & 0.82 & 1.88 & $2.599(2)$ & 146 \\
$\mathrm{O} 3-\mathrm{H} 3 \cdots \mathrm{O} 9$ & 0.82 & 1.71 & $2.5269(17)$ & 171 \\
$\mathrm{O} 8-\mathrm{H} 8 \cdots{ }^{\mathrm{i}}$ & 0.82 & 1.89 & $2.6979(18)$ & 171 \\
$\mathrm{O} 9-\mathrm{H} 1 W \cdots{ }^{\mathrm{ii}}$ & 0.84 & 1.93 & $2.753(2)$ & 165 \\
$\mathrm{O} 9-\mathrm{H} 2 W \cdots \mathrm{O} 5^{\mathrm{iii}}$ & 0.83 & 1.89 & $2.713(2)$ & 172 \\
$\mathrm{O} 10-\mathrm{H} 3 W \cdots \mathrm{O} 2$ & 0.81 & 2.32 & $2.9001(19)$ & 129 \\
$\mathrm{O} 10-\mathrm{H} 4 W \cdots \mathrm{O} 7^{\mathrm{iv}}$ & 0.81 & 2.27 & $2.835(2)$ & 128 \\
$\mathrm{~N} 2-\mathrm{H} 2 D \cdots \mathrm{O}^{\mathrm{i}}$ & 0.86 & 1.86 & $2.689(2)$ & 162 \\
\hline
\end{tabular}

Symmetry codes: (i) $-x+1,-y,-z$; (ii) $x, y+1, z$; (iii) $-x,-y+1,-z+1$; (iv) $x, y+1, z+1$; (v) $-x+1,-y+2,-z+1$. 